\title{
Ueber das Eindringen von Albumin in Gelatinegallerten.
}

\author{
Von G. Möllh a usen.
}

Eine Erscheinung, welche für die Deutung einiger biologischer Vorgänge von Bedeutung sein dürfte, tritt mit ziemlicher Regelmäßigkeit ein, wenn man über Gelatinegallerte trocknes Ei- oder Blutalbumin schichtet: Das Albumin sucht sich einen Weg in die Gallerte: Nach mehrtägigem Stehen zeigt sich ein Spalt in der letzteren. Zuweilen ist der Spalt schon nach einem Tag $5 \mathrm{~cm}$ tief und der năchste $\mathrm{Tag}$ kann eine gleiche weitere Verlängerung desselben bringen. Das Albumin hat inzwischen ganz unerwartet große Wassermengen der Gallerte entzogen und füllt den Spalt aus.

Die Versuche wurden angestellt mit Gallerten, welche aus $2-10$ prozentigen Gelatinelösungen bereitet worden waren. Diẹse waren in Reagensröhren erstarrt. Die Menge des aufgeschichteten trocknen Albumins war 2-5 g. Die Bildung des Spaltes; welcher meist mehrfach gewunden ist, erfolgt zumeist schon nach einem Tag, zuweilen aber erst nach mehreren Tagen. Bei einer Versuchsreihe von 26 Prăparaten, welche in einem Raum von $8^{\circ} \mathrm{C}$ standen, hatten nach 10 Tagen 16 Präparate noch keine Spaltung gezeigt. Als diese in einen Raum von $16^{\circ} \mathrm{C}$ gebracht wurden, zeigten schon nach zwei Stunden weitere 12 die Spaltung. - Eine höhere Temperatur ist dazu also günstig.

Zusatz von Phenol zur Gallerte oder zum Eiweib hindert die Spaltbildung nicht.

Die unerwartet große Wasseranziehung des Albumins aus der Gallerte wurde nichí ver- hindert, wenn der letzteren reichlich Salze zugesetzt wurden.

Bei einem Versuch war die Gallerte bereitet worden aus $2 \mathrm{~g}$ Gelatine, $2 \mathrm{~g}$ Bromkalium. $98 \mathrm{~g}$ Wasser. Die nach dem Erstarren aufgesetzten $3 \mathrm{~g}$ Eialbumin waren vorher in der gleichen Menge Wasser gelöst worden. Trotzdem trat die Wasseranreicherung ein und nach 36 Stunden auch die Spaltbildung.

Ebenso erstaunlich ist, daR die Einbettung von Albumin in die Gallerte die Spaltbildung durch aufgesetztes Albumin nicht hindert.

In eine Gallerte aus $2 \mathrm{~g}$ Gelatine, $10 \mathrm{~g} \mathrm{Ej}$ albumin (trocken), $88 \mathrm{~g}$ Wasser hatten sich ebenfalls nach 36 Stunden die überschichteten $3 \mathrm{~g}$ Eialbumin (gelöst in $3 \mathrm{~g}$ Wasser) spaltartig eingefressen.

Das Eindiffundieren von Bestandteilen des verwendeten Albumins in die Gallerte, seines Salzgehaltes und des gelben Farbstoffs geht der Spaltbildung vorher. Die Vermutung, daß letztere eine reine Gerbewirkung sei (es war dabei vorübergehend an die Möglichkeit einer Rückbildung von Kollagen aus der Gelatine gedacht worden), gab Veranlassung zu Kontrollversuchen mit Gerbemitteln. Aber weder Eisenoxydsalze, Chromalaun. Kalialaun, Tannin. Formaldehyd vermochten Spalten zu erzeugen. Auch übergeschichteter Alkohol nicht.

Dagegen wirkt Pepsin stärker spaltbildend als die bisher untersuchten Albuminate.

\section{Ueber blaues Erythrosinsilber.}

Von Dr. Lüppo-Cramer.

(Wissenschaftl. Laboratorium der Dr. C. Schleußner A.-G. zu Frankfurt a. M.)

Die Silbersalze der Eosinfarbstoffe fallen bekanntlich als unlösliche Niederschläge aus, wenn man die Alkalisalze der Farbsäuren mit Silbernitrat versetzt. Diese Silbersalze erhält man in schőnen kolloiden Lösungen, entweder wenn man Schutzkolloide, z. B. Gelatine, Gummi, bei der Ausfällung zugegen sein läBt, oder auch wenn man die Umsetzung in verdünnten Lossungen und bei Vermeidung eines Silbersalzüberschusses vornimmt. Man erhält besonders beim Erythrosin ein sehr feines Sol des Silbersalzes, das sich augenfällig von der Lösung des Natriumsalzes dadurch unterscheidet, daß die Lösung auBerordentlich viel intensiver gefärbt und ganz beträchtlich mehr nach blau nuanciert ist.

Als kolloider Körper ist das Erythrosinsilber in ähnlicher Weise einer Re if ung zugänglich wie die Silberhaloide. Schon bei kurzem Kochen einer verdünnten Lösung von Erythrosinsilber wird es opalisierend und nimmt eine immer mehr nach blau übergehende Farbe an. Schöner verlăuft diese Reifung bei ruhigem Stehen der kolloiden Lösung bei Zimmertemperatur. Nach einigen Tagen hat sich ein sehr feiner Bodensatz gebildet, der, von der überstehenden Lösung getrennt. in der Durchsicht eine rein blaue Farbe zeigt. Noch vollkominener vollzieht sich 
die Bildung eines ganz rein blau gefärbten Erythrosinsilbers, wenn man Brom-oder Chlorsilber gewissermaken als Schutzkolloide in der reifenden Lösung $\mathrm{zu}^{-}$ gegen sein läßt, so z. B. in folgender Weise: $1 \mathrm{~g}$ Erythrosin, gelöst in $400 \mathrm{ccm}$ Wasser, hierzu $7,1 \mathrm{ccm} 10$ prozentige $\mathrm{KBr}-\mathrm{Lösung}$, dann $12 \mathrm{ccm}$ 10 prozentige Silbernitratlösung.

Es entsteht hierbei zunächst keinerlei Trübung, sondern $\mathrm{AgBr}$, und Erythrosinsilber üben gegenseitig Schutzwirkung aus. ${ }^{1}$ )

Im Verlaufe von $2-3$ Tagen wird die ursprünglich ganz klare Lösung allmählich trübe und zeigt im reflektierten Lichte eine gelbrote Farbe. Der sich langsam bildende Bodensat? ist in der Durchsicht rein tiefblau gefärbt, er verteilt sich in Wasser zu einer feinen Suspension, die sich durch Papier filtrieren läbt, ohne einen Rückstand zu lassen. Bei mikroskopischer Betrachtung (Oel-Immersion) zeigt sich das gereifte Erythrosinsilber als Suspension von kleinen blau gefärbten Teilchen. Bei den

1) Lappo-Cramer, Photographische Probleme, Halle 1907, S. 26 a. f. Diese Zeitschr. 1, 227. vorzüglichen Eigenschaften der Eosinfarbstoffe als optischer Sensibilisatoren für die Silberhaloide lieb sich vermuten, dab auch das gereifte Erythrosinsilber, dessen Absorptionsband bis ins Orange gehts gut zu verwenden sein würde. Dies ist indessen nicht der Fall; wahrscheinlich sind die schon nicht mehr im Solzustande befindlichen Partikelchen des gereiften Farbstoffes nicht mehr imstande, die Silberhaloide anzufärben, so daß sich diese nur mit den in der Suspension noch vorhandenen ungereiften feineren Teilchen anfärben. Das mit dem blauen Erythrosinsilber behandelte Bromsilber zeigte nämlich keine merklich verschiedene Farbe gegenüber dem mit der frisch hergestellten Lösung gefärbten.

Die Verwandten des Erythrosins (Tetrajodfluoreszeinnnatrium), das Fluoreszeìn, Dibromfluoreszeïn, Tetrabromfluoreszeïn (Eosin) sowie Rose bengale (Tetrajoddichlorfluoreśzeïn) verhalten sich ganz anders als jenes: die kolloiden Lösungen der Silbersalze dieser Farbstoffe blieben in Gegenwart des kolloiden Bromsilbers wochenlang als ganz unveränderte Sole bestehen.

\section{Zur Lehre von den Zuständen der Materie.}

\section{(Fortsetzung.)}

Das Studium der Doppelumsetzungsreaktion in sehr konzentrierten Lösungen gestattet einige Schlüsse auf den Reaktionsverlauf in heterogenen Medien $z u$ ziehen und sogar eine bindende Brücke von der Kinetik homogener Systeme zur Kinetik heterogener Systeme zu schlagen.

Die Geschwindigkeit der Doppelumsetzung in konzentrierten Lösungen wird stark verzögert und verläuft sogar bej dem stärksten mechanischen Umrühren nicht bis zu Ende.

Dies wird durch die Eigenartigkeit der Niederschlagsformen bei groben Konzentrationen hervorgerufen, und die dabei auftretenden Erscheinungen verdienen eine besondere, aufmerksame Betrachiung.

Von einer bestimmten Konzentration der reagierenden Lösungen an kann man bemerken. daß die zugegossenen Tropfen der Lösung sich s of ort mit einem Ueberzug aus Niederschlag gewöhnlicher Form und mikrokristallinischer Struktur bedecken.

Für die Bildung eines solchen Ueberzuges ist es freilich notwendig, dab die Reaktion in einer gewissen Zone der sich berühren- den Lösungen von der Dicke $\triangle$ verläuft. Bei verhältnismäßig kleinen Konzentrationen ist $\Delta$ relativ groß, deshalb verläuft die Reaktion bis zu dem üblichen Ende und in den Zellen befindet sich keine noch nichi umgesetzte Lösung. Die Zellen sind außerdem spröde, zerfallen von selbst, und erleichtern dadurch den weiteren Verlauf der Reaktion, die deshalb nur wenig verzögert wird.

Je größer aber die Konzentration ist, um so verschwindend kleiner erscheint $\Delta$ und sowohl in den Zellen, als auch außerhalb derselben bleiben immer mehr und mehr konzentrierte, nöh nicht umgesetzte Lösungsteile zurück.

Bei den maximalen Konzentrationen ist $\Delta$ sehr klein, denn die Tropfen bedecken sich augenblicklich mit einem wasserhellen Ueberzug, dessen Durchlässigkeit für die verschjedenen Arten reagierender Moleküle praktisch Null ist.

In einer auf diese Weise entstandenen großzelligen Gallerte verläuft die Reaktion nach mehreren Stunden und sogar Tagen und Wochen nicht bis zu Ende, wenn die Gallerte sich selbst überlassen wird. Die Menge des in Form von 